E3S Web of Conferences 1, 09002 (2013)

DOI: $10.1051 / \mathrm{e} 3$ sconf/20130109002

(c) Owned by the authors, published by EDP Sciences, 2013

\title{
Liquid phase micro-extraction: Towards the green methodology for ultratrace metals determination in aquatic ecosystems
}

\author{
J. A. López-López ${ }^{1}$, C. Vergel, C. Mendiguchía, J. J. Pinto, V. Manuel, M. Silva, M. García-Vargas and C. Moreno
}

${ }^{1}$ University of Cádiz. Department of Analytical Chemistry. Faculty of Marine and Environmental Sciences. República

Saharaui s/n PC: 11510, Puerto Real (Spain), joseantonio.lopezlopez@uca.es

\begin{abstract}
Heavy metals are normally found, in natural waters, in very low concentrations. Some of them are essential for life in low level; however, in higher level they are toxic. Therefore, analyzing their bio-available fraction is of main interest. Standard methodology is based in the collection of a number of samples from a water body. Collected samples must be stored, pre-treated and then analyzed. Pre-treatment usually involves pre-concentrating the metal, with the corresponding risk of contamination or loss of analyte. This way, punctual information is obtained from every sampling campaign. As an alternative, passive sampling techniques allow the continuous and coupled sampling-pre-treatment for heavy metals analysis, giving a better approach in the characterization of the studied water body. Liquid phase micro-extraction (LPME) is a green analytical alternative for liquid-liquid extraction that promotes a reduction of sample volume, solvent needed and waste generation. Using these systems, polypropylene hollow fibers (HF) with pores in their walls can be used. A few micro-liters of organic solvent are supported in the pores. The sample is placed in the outer part of the fiber and a receiving phase is placed in its inner part, allowing continuous liquid extraction of the metal from the sample. Several fibers with different physical features have been employed to analyzed total concentration and bio-availability of some heavy metals $(\mathrm{Ag}, \mathrm{Ni}, \mathrm{Cu})$ in natural water samples. Thanks to fibers configuration, devices for passive sampling based in HF-LPME could be designed. Advantages of this methodology over existing ones are supported because the receiving phase is liquid. As a consequence, retained metals do not need to be eluted from the acceptor prior to instrumental analysis.
\end{abstract}

Key words: Heavy metals, liquid phase micro-extraction, speciation, passive sampling

\section{Introduction}

Although they are normally found at very low concentrations in surface waters, heavy metals are recognized as important pollutants of natural waters. Some of them are essential for life but in higher level they can be toxic. Therefore, the determination of heavy metals in the ultra-trace level is an important task for environmental analysis. In particular, their bio-available fraction must be determined for a better understanding of heavy metals uptake.

Most environmental monitoring programs are based in the collection of discrete samples. Moreover, big volumes of samples are needed because analytes are at very low concentration and need to be pre-concentrated for their determination. This way, analyzed concentrations give punctual information [1]. Therefore, for continuous monitoring frequency of sampling should be increased. As a consequence, collected samples have to be stored, pre-treated and analyzed, with the subsequent cost of analysis and wastes generation.

With this in mind, liquid phase micro-extraction (LPME) appears as a simple and environmentally friendly alternative for heavy metals pre-concentration. It needs fewer amount of sample than standard techniques. Moreover, only a few micro-liters of organic solvent are used. Additionally, they could potentially be applied for passive sampling in field studies. This way the analyte could be pre-concentrated in situ and the risk of sample contamination or the loss of analyte could be avoided.

LPME can be carried out using a hollow fiber (HF) as a support for the organic solution. The sample is placed outside the fiber while the receiving phase is in its inner part. Fiber walls consist in polymeric material 
where pores are impregnated with an organic solution that makes the contact between the sample and the receiving phase (Fig 1). Organic phase contains an organic ligand that transports the metal form the sample to a receiving solution.

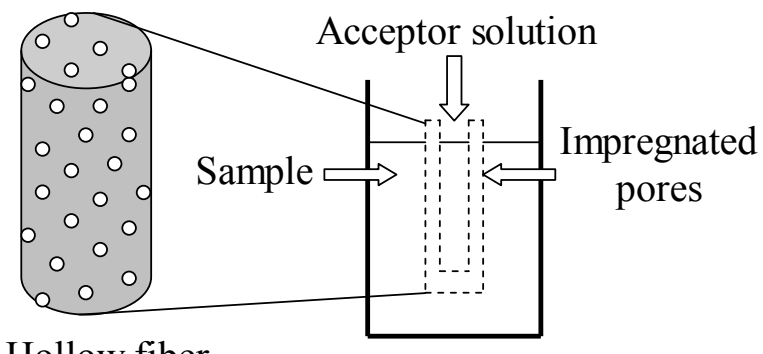

Hollow fiber

Fig. 1. Scheme of a hollow fiber used as support for liquid phase micro-extraction.

Advantages of HF-LPME against other passive sampling techniques as diffusive grandient in thin films (DGT) or Chemcatcher ${ }^{\circledR}$ are associated to its configuration. Hollow fibers present better tuning capability because all phases are liquids and reagents concentrations can be optimized in all of them. The most important feature is that the receiving phase is an aqueous solution. This avoids the needing of eluting the metal from the acceptor, as it happens with DGT or Chemcatcher ${ }^{\circledR}[2]$.

In the present work the use of LPME is exposed as an analytical tool for analyzing the bioavailable fraction of heavy metals in natural waters. Their use for silver, nickel, and copper analysis in natural waters is shown. Moreover, the potential of hollow fibers as passive sampling devices as a support for LPME in field studies is considered.

\section{Materials and Methods}

\section{Materials and reagents}

Hollow fibers were obtained from Membrana (Germany). Accurel PP 50/280 was used. This fiber has an internal diameter of $280 \mu \mathrm{m}$ and an average pore size of $0.1 \mu \mathrm{m}$. Hollow fiber Q3/2 has a pore size of $0.2 \mu \mathrm{m}$ and $600 \mu \mathrm{m}$ internal diameter. Finally, $\mathrm{S} 6 / 2$ pore size is $0.55 \mu \mathrm{m}$ and internal diameter is $1800 \mu \mathrm{m}$.

Different organic ligands were used for facilitated transport of the metal ions from the sample to the receiving phase. In the case of silver, tri-isobutyl phosphine sulphide (TIBPS) was used. Dietilhexyl phosphoric acid (DEHPA) and 1,2-ciclohexyl bis-benzoil hydrazone $(\mathrm{CHBBH})$ were used in the analysis of total concentration of nickel and for its speciation respectively. For copper analysis, di-2-pyridyl ketone benzoyl hydrazone (DPKBH) was applied.

Heavy metals concentrations in the receiving phase were measured by atomic absorption spectroscopy by flame using a ContrAA atomic absorption spectrometer (Analytik Jena, Germany).

\section{Procedure}

Set up of the HF-LPME system depended on the type of fiber (Fig 2). In the case of Accurel PP 50/280, the lumen of the fiber was filled up with the receiving solution using a syringe. Following, the pores of the fiber were impregnated by soaking in an organic solution containing the carrier. The lumen was rinsed with the receiving phase. Finally, the extremes of the fiber were sealed with paraffin film and it was immersed in the sample.

Q3/2 set up was similar. Fiber impregnation and filling the lumen were conducted in the same way as for Accurel PP 50/280. However, the fiber was not freely immersed in the beaker containing the sample. It was kept in the sample hanging from a cap of sample container.

When Accurel fiber S 6/2 was used, the first step was prepared impregnating the pores of the fiber with organic solution containing the carrier. Secondly, fibers were hanged on with a plastic support in the form of a loop. Thus, the internal volume was filled with the receiving phase using a peristaltic pump. Finally, the fiber was hanged in the sample during the experiment time as for Accurel Q3/2.

In all cases, at the end of the experiment, the receiving solution was flushed from the fiber using a syringe and metal concentration was measured by atomic absorption spectroscopy.

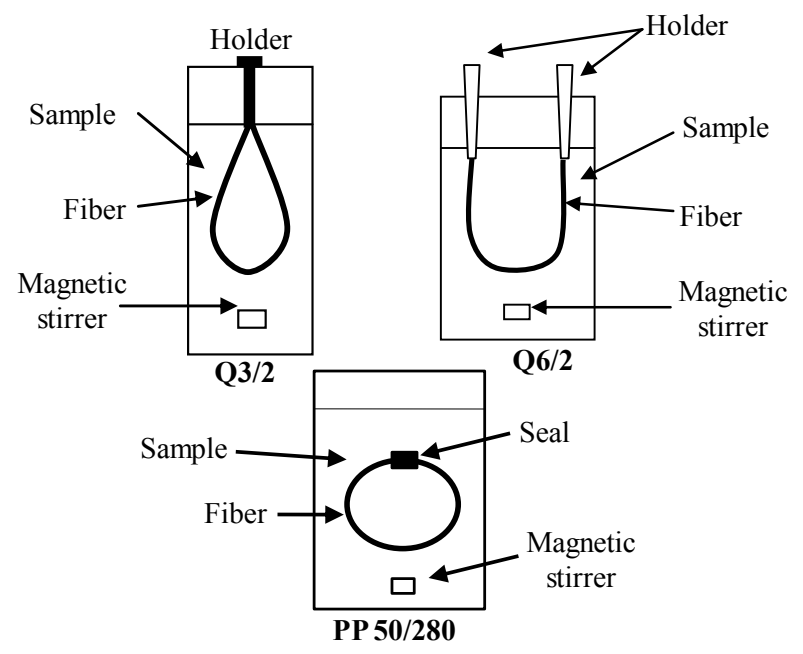

Fig 2. Set up of employed fibers

\section{Results and Discussion}

Hollow fiber liquid phase micro-extraction systems must be optimized considering two aspects. The first one is the design of the system, based on the physical characteristics of the fibers. In this sense, length of the fiber associated with its internal volume, pore size related with the impregnation procedure, and the possibility of 
automation were considered. The second one is related to the concentrations of the reagents used in all the liquid phases.

In the case of Accurel PP 50-280, impregnation of fiber pores was carried out once the fiber was filled with the receiving solution, to limit the pass of organic solvent inside the fiber. An important feature of this fiber is its small internal diameter $(280 \mu \mathrm{m})$. Thus, small volumes of receiving phase can be used, usually from 1 to $20 \mu 1$. As a consequence, on the one hand, higher volume ratios between the sample and the receiving phase can be obtained; on the other hand, manual procedures must be used to fill the fiber with the stripping solution.

As an example of Accurel PP 50/280 fiber application, total concentration of silver in river water has been analyzed. In order to transport $\mathrm{Ag}^{+}$, tri-isobutylphosphine sulphide (TIBPS) dissolved in dihexyl ether was used as carrier. Transport mechanism was based in the complexation of $\mathrm{Ag}^{+}$by TIBPS in the presence of $\mathrm{NO}_{3}^{-}$as a counter-anion, to form a ion pair that is soluble in the organic solvent. Finally, $\mathrm{Na}_{2} \mathrm{~S}_{2} \mathrm{O}_{3}$ was used as receiving agent in a volume of $20 \mu \mathrm{l}$. After the optimizing concentration of reagents in the aqueous phases and the membrane, enrichment factors about 1000 thousand times were obtained. This allowed the determination of silver in natural waters by graphite furnace atomic absorption at the ppt-levels.

Hollow fibers Q3/2 have been applied for the determination of total concentration of nickel in saline waters. The advantage of this fiber when compared with the previous one is its robutness. It has thicker walls and higher internal diameter. Ni transport was promoted by DEHPA dissolved in the organic phase by coupled counter-transport of $\mathrm{Ni}$ species and $\mathrm{H}^{+}$. Optimized variables were $\mathrm{pH}$ in the sample and concentrations of DEHPA and $\mathrm{HNO}_{3}$ in the organic phase and the acceptor solution respectively. Following, the system was applied for analyzing $\mathrm{Ni}$ in the level of $\mu \mathrm{g} \cdot \mathrm{L}^{-1}$ in estuarine samples from the Guadalquivir River estuary.

Finally, fiber Accurel S6/2 has been used in the transport of $\mathrm{Cu}$ by DPKBH dissolved in toluene and for $\mathrm{Ni}$ speciation in estuarine and seawater samples.

The mechanism of HF-LPME is considered by many authors to be similar to cellular membrane. This is supported because LPME allows the selective transport of the bio-available fraction of a metal from the sample [3]. For selective transport of bioavailable $\mathrm{Ni}, \mathrm{CHBBH}$ dissolved in toluene was used, and $\mathrm{HNO}_{3}$ was applied as acceptor agent. The system was compared with anodic voltammetry as a reference technique and results were in good agreement.

Physical features of Accurel S6/2 fiber make possible the automation of the set up process. Thus, a peristaltic pump was used to fill the lumen of the fiber with the acceptor solution. Advantages of automation are higher reproducibility and lower samples handling.

Attending to the results, LPME using HF supports, constitute an encironmentally friendly alternative for traditional liquid extraction techniques in the determination of the bio-available fraction of heavy metals.

Additionally to laboratory experiments, HF-LPME has potential applications in field studies. Existing passive samplers as DGT and Chemcatcher ${ }^{\circledR}$ retain metal ions in a polymeric material. Thus, the analyte must be eluted from the acceptor prior to instrumental determination. Elution is avoided in HF-LPME because the receiving phase is an aqueous solution.

\section{Conclusion}

Liquid phase micro-extraction, using hollow fibers as supporting material is an environmentally friendly alternative for the speciation of heavy metals in natural waters.

Selective transport of different fractions of the heavy metals from water samples can be carried out using organic ligands as carriers, and clean up of the sample and the analysis of heavy metals bio-availability can be conducted. Important pre-concentrations can be obtained and the enrichment factors can be easily tuned varying the fiber length or internal diameter.

Due to their physical properties, they appear as a potential alternative for in situ pre-treatment in environmental waters analysis.

\section{Acknowledgements}

This work has been supported by Spanish Ministerio de Economía y Competitividad (CTM2010-15618).

\section{References}

1. Seethapathy, S.; Górecki, T.;Li, X. Passive sampling in environmental analysis. Journal of Chromatography A. 2008; 1184:234-253.

2. Allan, I.J.; Knutsson, J.; Guigues, N.; Mills, G.A.; Fouillac, A and Greenwood, R. Chemcatcher ${ }^{\circledR}$ and DGT passive sampling devices for regulatory monitoring of trace metals in surface water. Journal of Environmental Monitoring 2008; 10:721-725

3. Slaveykova, V.; Parthasarathy, N.; Buffle, J., and Wilkinson, K.J. (2004) Permeation liquid membrane as a tool for monitoring bioavailable $\mathrm{Pb}$ in natural waters. Sci. of Tot. Environ. 328, 55-68 